\title{
Consolidação de Bases para o Diagnóstico do Distrito de Inovação de Blumenau
}

\author{
João Luiz Fernandes', Aurélio Faustino Hoppe ${ }^{1}$, Christian Krambeck ${ }^{1}$, Rosemeri \\ Laurindo $^{1}$, Julio Cesar Refosco ${ }^{1}$, Ralf Marcos Ehmke ${ }^{1}$ \\ Universidade Regional de Blumenau (FURB) \\ Rua Antônio da Veiga, 140 - 89030-903 - Blumenau - SC - Brasil \\ joaooluizzflgmail.com, \{aureliof, ckrambeck, rlaurindo, refosco, \\ ehmke\} afurb.br
}

\begin{abstract}
This paper presents the process of consolidating the databases of the Brazilian Institute of Geography and Statistics (IBGE), OpenStreetMaps (OSM) and the Municipality of Blumenau (PMB), with the purpose of making a diagnosis about the socioeconomic and developmental aspects of neighborhoods Victor Konder and Itoupava Seca of the city of Blumenau, being able to be adapted for the diagnosis of other regions. Such information is relevant to the understanding of the district and also considerable for the planning of policies and actions that may occur in the region considering five axes: city, people, environment, economy and mobility.
\end{abstract}

Resumo. Este artigo apresenta o processo de consolidação das bases de dados do Instituto Brasileiro de Geografia e Estatística (IBGE), OpenStreetMaps (OSM) e da Prefeitura Municipal de Blumenau (PMB), tendo como intuito fazer o diagnóstico quanto aos aspectos socioeconômicos e de desenvolvimento dos bairros Victor Konder e Itoupava Seca da cidade de Blumenau, podendo ser adaptado para o diagnóstico de outras regiões. Tais informações se mostram pertinentes para a compreensão do distrito e também consideráveis para o planejamento de políticas e ações que venham a ocorrer na região considerando cinco eixos: cidade, pessoas, ambiente, economia e mobilidade.

\section{Introdução}

Por muitos anos, a inovação estava fortemente relacionada aos produtos tangíveis das indústrias manufatureiras, os serviços adotavam essas inovações tecnológicas, mas produziam poucas inovações em seu próprio contexto [Kon 2016]. Esta perspectiva tradicional é questionada por estudos recentes, que identificaram a intensa inovação em atividades de serviços, inclusive em setores que não apresentam fins lucrativos, como nos setores de serviços sociais e públicos. Evoluímos de um modelo de desenvolvimento baseado na produção primária e na indústria, para uma nova economia, fundamentada na informação e no conhecimento, surgiram novos arranjos e ambientes de desenvolvimento, que substituíram os antigos distritos industriais e passaram a protagonizar o processo desenvolvimento econômico e social e de geração de emprego e renda [Audy e Piqué 2016]. 
Estes ambientes de inovação, ou ecossistemas de inovação como são conhecidos no Brasil, são uma realidade em vários países. Como exemplo têm-se os distritos 22@Barcelona e LxFactory em Lisboa. Em território nacional existem os distritos de Pedra Branca em Palhoça, join.vale em Joinville e o Distrito C em Porto Alegre.

Para [Komninos 2008], o que distingue os ecossistemas de inovação de outras regiões é sua capacidade de reforçar o desempenho de inovação das organizações que se estabeleceram no local. Em Santa Catarina, uma das estratégias vigentes é a implantação de treze centros de Inovação, inseridos de forma descentralizada em diferentes regiões do Estado. Sendo um deles em Blumenau, servindo como pilar para ativar o ecossistema de inovação, ser referência em apoio ao empreendedorismo inovador e ser o motor da cultura inovadora [Teixeira et al. 2016]. Além disso, em 2017 o Governo do Estado publicou o Guia de Implantação dos Centros de Inovação, que apresenta os conceitos, fundamentos e diretrizes para a instalação dos Centros nas regiões catarinenses. No entanto, o guia pode servir para a implementação de qualquer habitat de inovação, já que oferece portfólios de soluções que podem ser customizados conforme a realidade de cada local [Governo do Estado de Santa Catarina 2017].

Diante do exposto, este trabalho apresenta o processo de consolidação das bases de dados do Instituto Brasileiro de Geografia e Estatística (IBGE), OpenStreetMaps (OSM) e da Prefeitura Municipal de Blumenau (PMB). Visando estabelecer um diagnóstico socioeconômico e de desenvolvimento dos bairros Victor Konder e Itoupava Seca da cidade de Blumenau, local onde será implantado o Centro de Inovação dentro do município. Tendo como intuito viabilizar a criação de um distrito de inovação na região. Os indicadores gerados também poderão ser utilizados como base para criação do Plano Estratégico de Desenvolvimento Econômico Municipal de Blumenau (PEDEN), e para avaliar o impacto das políticas sociais que serão tomadas ou negligenciadas nos próximos anos. O diagnóstico é apresentado através de um Sistema de Informação Geográfica (SIG), cujo objetivo é apoiar a manipulação, análise e visualização dos dados geográficos assim como foi feito por [Azevedo 2005].

\section{Coleta, estruturação e análise dos dados}

Os dados do IBGE foram obtidos através do seu portal para download. Foram coletados os dados dos censos de 2010, agregados por setores censitários e suas respectivas malhas digitais. Os dados são disponibilizados por unidade da federação, tanto em formato CSV quanto XLS, junto aos dados é necessário realizar o download da documentação contendo a explicação para cada variável das tabelas. Quanto aos dados do OSM, utilizou-se um plugin da ferramenta ArcGis. No OSM os Elementos são os componentes básicos para a reprodução do mundo, eles consistem de Nós, Caminhos e Relações, ambos podem ser associados a uma ou mais Tags, as quais descrevem significados para os elementos. Por fim, os dados da PMB, foram obtidos através de contato da FURB com a Prefeitura, foram obtidos os dados referentes a todo o município do ano de 2003 e fotos aéreas das regiões do Victor Konder e Itoupava Seca. Também foram obtidos dados do Plano Mobilidade da cidade, contendo dados sobre o transporte público, ciclovias e ciclo faixas existentes e que serão implementadas e o cadastro de lotes de 2018 para a região. 
Após a coleta e análise dos dados, foi realizado a consolidação das bases utilizando o software ArcGis, pois possui todos os recursos necessários para manipulação, análise e visualização das informações. Outro ponto importante é que os dados da PMB já estavam no formato da ferramenta, além disso ela possui o plugin para integração com os dados do OSM. Após a importação dos dados, uniformizou-se o sistema de coordenadas geográficas utilizados pelas diferentes bases. Inicialmente cada uma das bases possuía um sistema de coordenadas, foi aplicado o sistema vigente para os dados da prefeitura em todas as bases, sendo ele o SAD 1969 UTM Zone 22S. Também se realizou a atualização de Tags do OSM que seriam utilizadas na geração de mapas, vinculando os edifícios do OSM e da PMB. Na sequência, foi gerado um File Geodatabase com os arquivos consolidados das diferentes bases.

A partir desse processo, foi possível realizar a geração dos indicadores utilizados para o diagnóstico do distrito. Foram utilizados os eixos definidos no ranking European Smart Cities: economia, pessoas, governança, mobilidade, ambiente e cidade. Os mapas foram gerados em formato JPEG e PDF, para os mapas gerados, foi mantido um arquivo de projeto, com a extensão .mxd, que permite a alteração e a geração de um novo mapa. A figura 1 traz os mapas de potencial construtivo (A) e cotas de enchente (B) gerados respectivamente para os eixos cidade e ambiente.

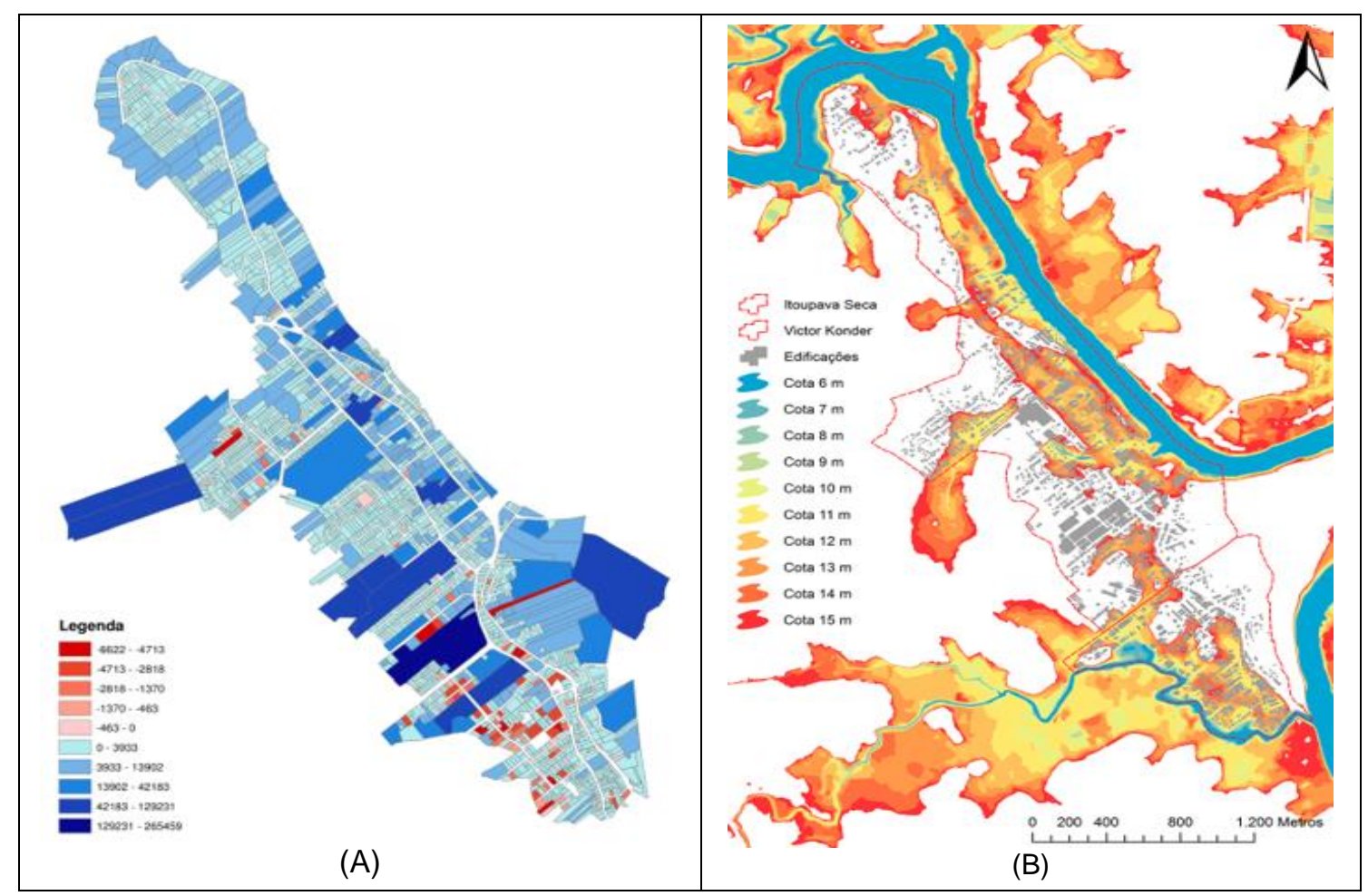

Figura 1. Mapas de Potencial construtivo (A) e Cotas de Enchente (B)

A partir do mapa de potencial construtivo (eixo cidade), foi calculado que o distrito possui hoje um total de 1 milhão de metros quadrados construídos. E possui ainda uma área de 5 milhões de metros quadrados que podem ser construídos, de acordo com o plano diretor vigente, representando um potencial de 5 vezes a área atual. No mapa, é possível observar que as áreas em azul representam o potencial construtivo, sendo as áreas mais intensa as de maior potencial. Já os trechos em vermelho, ultrapassam o limite estabelecido no plano diretor, mas não necessariamente estão fora 
da legislação. Através do mapa que aponta as cotas de enchentes (eixo ambiente), foi levantado que a partir da cota de 10 metros, 18 ruas são atingidas, com a cota de 12 metros, 72 ruas são atingidas e com a cota de 14 metros, 106 são alagadas, um total de $77 \%$ da área, o que representa um dos desafios para construção do distrito. No mapa é possível observar as áreas livres de enchentes, mais indicadas para construção. E também as áreas onde será necessário lidar com os alagamentos. É importante destacar que a base desenvolvida também permita a geração de diferentes mapas não explorados nesse artigo, tais como renda per capita, população residente e densidade populacional, ocupação do solo, hierarquia viária, itinerário das linhas de ônibus, ciclovias, equipamentos urbanos (escolas, postos de saúde, assistência social, igrejas, museus), indústrias, comércios, estacionamentos, classificação das calçadas, entre outros.

\section{Considerações finais}

É possível concluir que a consolidação das bases de dados do Instituto Brasileiro de Geografia e Estatística (IBGE), OpenStreetMaps (OSM) e da Prefeitura Municipal de Blumenau (PMB), se mostrou uma fonte notável para o diagnóstico de aspectos socioeconômicos e de desenvolvimento. Através da base consolidada é possível diagnosticar aspectos de diferentes eixos, como cidade, pessoas, ambiente, economia, mobilidade, entre outros. As informações obtidas se mostram pertinentes para a compreensão do distrito e também consideráveis para o planejamento de políticas e ações que venham a ocorrer na região. A metodologia utilizada para a criação da base se mostra válida não apenas para os bairros do distrito, mas pode ser aplicada a qualquer outra região a fim de possibilitar o diagnóstico. A escolha da ferramenta ArcGis para a manipulação dos dados se destacou pela facilidade na integração das bases e visualização das informações, apesar disso, se mostrou necessário um analista com conhecimento na ferramenta para operacionalizar a criação dos mapas.

\section{Referências}

Audy, J., Piqué, J. Dos Parques Científicos e Tecnológicos aos Ecossistemas de Inovação. Brasilia: Anprotec, 2016. 26 p.

Azevedo, J. et al. Proposta metodológica para análise de dados socioeconômicos e ambientais para planejamento e definição de políticas públicas. Cadernos Ebape.br, [s.1.], v. 3, n. 4, p. 1-12, dez. 2005. FapUNIFESP (SciELO).

Governo do Estado de Santa Catarina. Secretaria de Estado do Desenvolvimento Econômico Sustentável. Guia de Implantação dos Centros de Inovação: Livro Iconceito e fundamentos. Florianópolis: Governo de Santa Catarina, 2017. 74 p.

Komninos, N. Intelligent Cities and Globalisation of Innovation Networks. New York: Routledge, 2008. 307 p.

Kon, A. Ecossistemas de inovação: a natureza da inovação em serviços. Revista de Administração, Contabilidade e Economia da Fundace, [s.1.], v. 7, n. 1, p. 14-27, 11 mar. 2016.

Teixeira, C., et al. Ecossistema de inovação na educação de Santa Catarina. Vieira, M. S.; Teixeira, C. S. T.; Ehlers, A. C.T.(Orgs). Educação fora da caixa, v. 2, p. 11-30, 2016. 